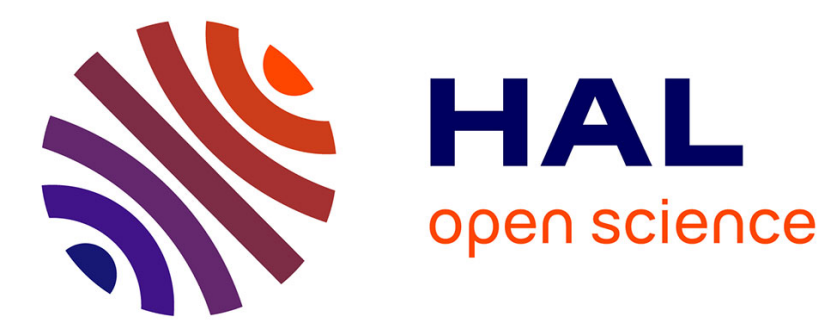

\title{
Hedging of American Options under Transaction Costs
}

Dimitri de Vallière, Emmanuel Denis, Yuri Kabanov

\section{To cite this version:}

Dimitri de Vallière, Emmanuel Denis, Yuri Kabanov. Hedging of American Options under Transaction

Costs. Finance and Stochastics, 2009, 13 (1), pp.105-119. hal-00700837

\section{HAL Id: hal-00700837 \\ https://hal.science/hal-00700837}

Submitted on 24 May 2012

HAL is a multi-disciplinary open access archive for the deposit and dissemination of scientific research documents, whether they are published or not. The documents may come from teaching and research institutions in France or abroad, or from public or private research centers.
L'archive ouverte pluridisciplinaire HAL, est destinée au dépôt et à la diffusion de documents scientifiques de niveau recherche, publiés ou non, émanant des établissements d'enseignement et de recherche français ou étrangers, des laboratoires publics ou privés. 
Dimitri De Vallière · Emmanuel Denis •

Yuri Kabanov

\title{
Hedging of American Options under Transaction Costs
}

Received: date / Accepted: date

\begin{abstract}
We consider a continuous-time model of financial market with proportional transaction costs. Our result is a dual description of the set of initial endowments of self-financing portfolios super-replicating Americantype contingent claim. The latter is a right-continuous adapted vector process describing the number of assets to be delivered at the exercise date. We introduce a specific class of price systems, called coherent, and show that the hedging endowments are those whose "values" are larger than the expected weighted "values" of the pay-off process for every coherent price system used for the "evaluation" of the assets.
\end{abstract}

Keywords Transaction costs · American option · Hedging · Coherent price system

Mathematics Subject Classification (2000) 91B28 · 60G42

\section{JEL Classification G10}

D. De Vallière

Laboratoire de Mathématiques, Université de Franche-Comté, 16 Route de Gray, 25030 Besançon, cedex, France

E-mail: dimitri.devalliere@univ-fcomte.fr

E. Denis

Laboratoire de Mathématiques, Université de Franche-Comté, 16 Route de Gray, 25030 Besançon, cedex, France

E-mail: emmanuel.denis6@wanadoo.fr

Y. Kabanov

Laboratoire de Mathématiques, Université de Franche-Comté, 16 Route de Gray, 25030 Besançon, cedex, France, and

Central Economics and Mathematics Institute, Moscow, Russia

E-mail: Youri.Kabanov@univ-fcomte.fr 


\section{Introduction}

A classical result of the theory of frictionless market asserts that the set of initial capitals needed to hedge a European option $\xi$ with the maturity (=exercise) date $T$ is a semi-infinite closed interval $\left[x_{*}, \infty[\right.$ whose left extremity $x_{*}=\sup _{\rho} E \rho_{T} \xi$ where $\rho=\left(\rho_{t}\right)$ runs through the set of martingale densities for the price process $S$. Recall that "to hedge" means to dominate the random variable $\xi$ by the terminal value of a self-financing portfolio. Basically, the assertion remains the same for the case of American-type option which pay-off is an adapted càdlàg stochastic process $f=\left(f_{t}\right)_{t \leq T}$. In this case, $x_{*}=\sup _{\rho, \tau} E \rho_{\tau} f_{\tau}$ where $\tau$ (an exercise date) runs through the set of stopping times dominated by $T$. "To hedge" means here to dominate, on the whole time interval, the pay-off process by a portfolio process. In both cases, as was shown by Dmitri Kramkov [12], the results can be deduced from the optional decomposition theorem applied to a corresponding Snell envelope.

We deliberately formulated the statements above (omitting assumptions) in terms of density processes rather than in terms of martingale measures to facilitate the comparison with the corresponding theorems for models with market friction.

In the theory of markets with transaction costs hedging theorems for European options are already available for discrete-time as well as for continuoustime models. Mathematically, in discrete-time, the model is given by an adapted cone-valued process $G=\left(G_{t}\right)_{t=0,1, \ldots, T}$ in $\mathbf{R}^{d}$. The portfolio (value) process $X$ is adapted and its increments $\Delta X_{t}=X_{t}-X_{t-1}$ are selectors of the random cones $-G_{t}$. The contingent claim $\xi$ is a random vector. The hedging problem is to describe the set $\Gamma$ of initial values $x$ for which one can find a value process $X$ such that $x+X_{T}$ dominates $\xi$ in the sense of the partial ordering induced by the cone $G_{T}$. It happens that, under appropriate assumptions,

$$
\Gamma=\left\{x \in \mathbf{R}^{d}: Z_{0} x \geq E Z_{T} \xi \quad \forall Z \in \mathcal{M}_{0}^{T}\left(G^{*}\right)\right\}
$$

where $\mathcal{M}_{0}^{T}\left(G^{*}\right)$ is the set of martingales evolving in the (positive) duals $G_{t}^{*}$ of the cones $G_{t}$. In the financial context, $G_{t}$ are solvency cones $\widehat{K}_{t}$, "hat" means that the assets are measured in physical units (the notation $K_{t}$ is used for the solvency cones when values of assets are expressed in units of a numéraire), and the elements of $\mathcal{M}_{0}^{T}\left(\widehat{K}^{*}\right)$ are called consistent price systems. For the continuous-time model the description remains the same but the theorem becomes rather delicate. The reason for this is that the model formulation is more involved and even the basic definition of value processes has several versions. Moreover, one needs assumptions on the regularity of the conevalued process, see the development and extended discussion of financial aspects in [5], [8], [10], [11], [3].

The hedging problem for the vector-valued American option $U=\left(U_{t}\right)$ in the discrete-time framework with transaction costs was investigated in the paper [2] by Bruno Bouchard and Emmanuel Temam (see also the earlier article [4] where the two-asset case for finite $\Omega$ was studied). It happens that one needs a richer set of "dual variables" to describe the set $\Gamma$ formed by the initial values of self-financing portfolios dominating, in the sense of partial 
ordering, the vector-valued adapted pay-off process $U$. Bouchard and Temam proved the identity

$$
\Gamma=\left\{x \in \mathbf{R}^{d}: \bar{Z}_{0} x \geq E \sum_{t=0}^{N} Z_{t} U_{t} \quad \forall Z \in \mathcal{Z}_{d}\left(G^{*}, P\right)\right\}
$$

where $\mathcal{Z}_{d}\left(G^{*}, P\right)$ is the set of discrete-time adapted process $Z=\left(Z_{t}\right)$ such that the random variables $Z_{t}, \bar{Z}_{t} \in L^{1}\left(G_{t}^{*}\right)$ for all $t \leq T$ with the notation $\bar{Z}_{t}:=\sum_{s=t}^{T} E\left(Z_{s} \mid \mathcal{F}_{t}\right)$. Note that the inclusion $\mathcal{M}_{0}^{T}\left(G^{*}\right) \subseteq \mathcal{Z}_{d}\left(G^{*}, P\right)$ is obvious.

In the theory of financial markets with transaction costs modelling of portfolio processes is rather involved. It is quite convenient to consider rightcontinuous portfolio processes and work in the standard framework of stochastic calculus. This approach leads to satisfactory hedging theorems, e.g., for a model with constant transaction costs and a continuous price process, see [8], [10], [11]. However, as was shown by Miklós Rásonyi in [13], such a definition is not appropriate when the price process is discontinuous: in general, the natural formulation of the hedging theorem (for European options) fails to be true. Luciano Campi and Walter Schachermayer in [3] suggested a more complicated definition of the portfolio processes for which the natural formulation of hedging theorem can be preserved.

In the present note we investigate the hedging problem using the approach of Campi and Schachermayer in a slightly more general mathematical framework. This framework is described in the next section where some basic concepts are introduced. In Section 3 we recall the definition of portfolio processes together with some known results adjusted to our purposes and accompanied by explicative comments. Section 4 contains the formulation of the main theorem preceding by a discussion of objects involved. Financial interpretation is given in the concluding Section 5 .

\section{Basic Concepts}

Standing hypotheses. We shall work from the very beginning in a slightly more general and more transparent "abstract" setting where we are given two cone-valued processes $G=\left(G_{t}\right)_{t \in[0, T]}$ and $G^{*}=\left(G_{t}^{*}\right)_{t \in[0, T]}$ in duality, i.e. $G_{t}^{*}(\omega)$ is the positive dual of the cone $G_{t}(\omega)$ for each $\omega$ and $t$. We suppose that $G_{t}=$ cone $\left\{\xi_{t}^{k}: k \in \mathbf{N}\right\}$ where the generating processes are càdlàg, adapted, and for each $\omega$ only a finite number of vectors $\xi_{t}^{k}(\omega), \xi_{t-}^{k}(\omega)$ are different from zero, i.e. the cones $G_{t}(\omega)$ and $G_{t-}(\omega):=\operatorname{cone}\left\{\xi_{t-}^{k}(\omega): k \in \mathbf{N}\right\}$ are polyhedral, hence, closed.

Throughout the paper we assume that all cones $G_{t}$ contain $\mathbf{R}_{+}^{d}$ and are proper, i.e. $G_{t} \cap\left(-G_{t}\right)=\{0\}$ or, equivalently, int $G_{t}^{*} \neq \emptyset$; moreover, we assume that the cones $G_{t-}$ are also proper.

In a more specific financial setting (see [11], [3]) the cones $G_{t}$ are the solvency cones $\widehat{K}_{t}$ provided that the portfolio positions are expressed in physical 
units $^{1}$. The hypothesis that the cones $G_{t}$ are proper means that there is efficient friction.

It is important to note that, in general, the continuity of generators does not imply the continuity of the cone-valued processes. The following simple example in $\mathbf{R}^{2}$ gives an idea: the process $G_{t}=\operatorname{cone}\left\{\xi_{t}^{1}, \xi_{t}^{2}\right\}$ where $\xi_{t}^{1}=e_{1}$, $\xi_{t}^{1}=(t-1)^{+} e_{2}$ is not right-continuous though the generators are continuous.

To formulate the needed regularity properties of $G$ we introduce some notation. Let $G_{s, t}(\omega)$ denote the closure of cone $\left\{G_{r}(\omega): s \leq r<t\right\}$ and let

$$
G_{s, t+}:=\cap_{\varepsilon>0} G_{s, t+\varepsilon}, \quad G_{s-, t}:=\cap_{\varepsilon>0} G_{s-\varepsilon, t}, \quad G_{s-, t+}:=\cap_{\varepsilon>0} G_{s-\varepsilon, t+\varepsilon}
$$

with an obvious change when $s=0$.

We assume that $G_{t, t+}=G_{t}, G_{t-, t}=G_{t-}$, and $G_{t-, t+}=\operatorname{cone}\left\{G_{t-}, G_{t}\right\}$ for all $t$.

It is easy to see that these regularity conditions are fulfilled for the case where the cones $G_{t}$ and $G_{t-}$ are proper and generated by a finite number of generators of unit length. Indeed, let $G_{t}=$ cone $\left\{\xi_{t}^{k}: k \leq n\right\}$ with $\left|\xi_{t}^{k}\right|=1$ for all $t$. Since the dependence on $\omega$ here is not important we may argue for the deterministic case. Let $x \notin G_{t}$. The proper closed convex cones $\mathbf{R}_{+} x$ and $G_{t}$ intersect each other only at the origin, so the intersections of the interiors of $\left(-\mathbf{R}_{+} x\right)^{*}$ and $G_{t}^{*}$ is non-empty (this is a corollary of the Stiemke lemma as it is given in the appendix in [9]). It follows that there is $y \in \mathbf{R}^{d}$ such that $x$ belongs to the open half-space $\{z: y z<0\}$ while the balls $\left\{z:\left|z-\xi_{t}^{k}\right|<\delta\right\}$ for sufficiently small $\delta>0$ lay in the complementary half-space. Since $\xi^{k}$ are right-continuous, the cones $G_{s, t+\varepsilon}$ for all sufficiently small $\varepsilon>0$ also lay in the latter. Thus, $x \notin G_{t, t+}$ and $G_{t, t+} \subseteq G_{t}$. The opposite inclusion is obvious. In the same way we get other two identities.

Example. Let us consider a financial market with constant proportional transaction costs given by a matrix $\Lambda=\left(\lambda^{i j}\right)$ defining the proper solvency cone $K$ (in terms of a numéraire). Suppose that the components of the positive càdlàg price process $S$ are such that $\inf _{t} S_{t}^{i}>0, i=1, \ldots, d$. Let us consider the mapping

$$
\phi_{t}:\left(x^{1}, \ldots, x^{d}\right) \mapsto\left(x^{1} / S_{t}^{1}, \ldots, x^{d} / S_{t}^{d}\right) .
$$

The generators of the cone $G_{t}=\widehat{K}=\phi_{t} K$ are vectors $\phi_{t} x_{i}$, where $x_{i}, i \leq N$, are generators of the polyhedral cone $K$ (they can be written explicitly in terms of $\Lambda$ ). The generators of the cone $G_{t}^{*}=\widehat{K}^{*}=\phi_{t}^{-1} K^{*}$ are vectors $\phi_{t}^{-1} z_{i}$, where $z_{i}, i \leq M$, are generators of the polyhedral cone $K^{*}$.

All above hypotheses are fulfilled for this model. Moreover, if $S$ admits an equivalent martingale measure with the density process $\rho$ and if $w \in \operatorname{int} K^{*}$, then the process $Z$ with the components $Z_{t}^{i}=w^{i} S_{t}^{i} \rho_{t}$ is a martingale such that $Z_{t} \in \operatorname{int} G^{*}$ and $Z_{t-} \in \operatorname{int}\left(G_{t-}\right)^{*}=\operatorname{int} G_{t-}^{*}$ for all $t$. Existence of such a martingale is the major assumption of the hedging theorem.

\footnotetext{
1 The notation $K_{t}$ is reserved for the solvency cones when the portfolio positions are expressed in terms of a numéraire.
} 
Remark. The argument above shows that the regularity assumptions hold for the model specified as in [3], i.e. for the case where $G_{t}=\widehat{K}\left(\Pi_{t}\right)$ and $G_{t-}=\widehat{K}\left(\Pi_{t-}\right)$ are proper cones generated by the bid-ask process $\Pi=\left(\Pi_{t}\right)$.

Comment on notation. As usual, $L^{0}\left(G_{t}, \mathcal{F}_{t}\right)$ is the set of $\mathcal{F}_{t}$-measurable selectors of $G_{t}, \mathcal{M}_{0}^{T}\left(G^{*}\right)$ stands for the set of martingales $M=\left(M_{t}\right)_{t \leq T}$ with trajectories evolving in $G^{*} ; \mathbf{1}:=\sum e_{i}=(1, \ldots, 1) ;\|Y\|_{t}$ is the total variation of the function $Y$ on the interval $[0, t]$.

Let $B$ be a càdlàg adapted process of bounded variation. We shall denote by $\dot{B}$ the optional version of the Radon-Nikodym derivative $d B / d\|B\|$ with respect to the total variation process $\|B\|$. In particular, this notation will be used for $B=Y_{+}$where $Y_{+}=\left(Y_{t+}\right)$.

We denote by $\mathcal{D}=\mathcal{D}(G)$ the subset of $\mathcal{M}_{0}^{T}\left(\operatorname{int} G^{*}\right)$ formed by martingales $Z$ such that not only $Z_{t} \in L^{0}\left(\operatorname{int} G_{t}^{*}, \mathcal{F}_{t}\right)$ but also $Z_{t-} \in L^{0}\left(\operatorname{int}\left(G_{t-}\right)^{*}, \mathcal{F}_{t}\right)$ for all $t \in[0, T]$. In the financial context the elements of $\mathcal{D}$ are called consistent price systems.

Coherent price systems. Let $\nu$ be a finite measure on the interval $[0, T]$ and let $\mathcal{N}$ denote the set of all such measures. For an $\mathbf{R}_{+}^{d}$-valued process $Z$ we denote by $\bar{Z}^{\nu}$ the optional projection of the process $\int_{[t, T]} Z_{s} \nu(d s)$, i.e. an optional process such that for every stopping time $\tau \leq T$ we have

$$
\bar{Z}_{\tau}^{\nu}=E\left(\int_{[\tau, T]} Z_{s} \nu(d s) \mid \mathcal{F}_{\tau}\right) .
$$

The process $\bar{Z}^{\nu}$ can be represented as a difference of a martingale and a left-continuous process whose components are increasing:

$$
\bar{Z}_{t}^{\nu}=E\left(\int_{[0, T]} Z_{s} \nu(d s) \mid \mathcal{F}_{t}\right)-\int_{[0, t[} Z_{s} \nu(d s) .
$$

We associate with $\nu$ the product-measure $P^{\nu}(d \omega, d t)=P(d \omega) \nu(d t)$ on the space $\left(\Omega \times[0, T], \mathcal{F} \times \mathcal{B}_{[0, T]}\right)$; the average with respect to this measure is denoted by $E^{\nu}$.

Let $\mathcal{Z}\left(G^{*}, P, \nu\right)$ denote the set of adapted càdlàg processes $Z \in L^{1}\left(P^{\nu}\right)$ such that $Z_{t}, \bar{Z}_{t}^{\nu} \in L^{0}\left(G_{t}^{*}, \mathcal{F}_{t}\right)$ for all $t \leq T$. We call the elements of this set coherent price systems. In the case where $Z$ is a martingale, $\bar{Z}_{\tau}^{\nu}=\nu([\tau, T]) Z_{\tau}$ and, hence, $\mathcal{M}_{0}^{T}\left(G^{*}\right) \subseteq \mathcal{Z}\left(G^{*}, P, \nu\right)$.

\section{The Model and Prerequisites}

We define the portfolio processes following the paper [3]. For the reader convenience, we give also full proofs of the basic properties.

Let $Y$ be a $d$-dimensional predictable process of bounded variation starting from zero and having trajectories with left and right limits (French abbreviation: làdlàg). Put $\Delta Y:=Y-Y_{-}$, as usual, and $\Delta^{+} Y:=Y_{+}-Y$ where $Y_{+}=\left(Y_{t+}\right)$. Define the right-continuous processes

$$
Y_{t}^{d}=\sum_{s \leq t} \Delta Y_{s}, \quad Y_{t}^{d,+}=\sum_{s \leq t} \Delta^{+} Y_{s}
$$


(the first is predictable while the second is only adapted) and, at last, the continuous one:

$$
Y^{c}:=Y-Y^{d}-Y_{-}^{d,+}
$$

Recall that $\dot{Y}^{c}$ denotes the optional version of the Radon-Nikodym derivative $d Y^{c} / d\left\|Y^{c}\right\|$.

Let $\mathcal{Y}$ be the set of such process $Y$ satisfying the following conditions:

1) $\dot{Y}^{c} \in-G d P d\left\|Y^{c}\right\|$-a.e.

2) $\Delta^{+} Y_{\tau} \in-G_{\tau}$ a.s. for all stopping times $\tau \leq T$;

3) $\Delta Y_{\sigma} \in-G_{\sigma-}$ a.s. for all predictable ${ }^{2}$ stopping times $\sigma \leq T$.

Let $\mathcal{Y}^{x}:=x+\mathcal{Y}, x \in \mathbf{R}^{d}$. We denote by $\mathcal{Y}_{b}^{x}$ the subset of $\mathcal{Y}^{x}$ formed by the processes $Y$ bounded from below in the sense of partial ordering, i.e. such that $Y_{t}+\kappa_{Y} \mathbf{1} \in L^{0}\left(G_{t}, \mathcal{F}_{t}\right), t \leq T$, for some $\kappa_{Y} \in \mathbf{R}$. In the financial context (where $G=\widehat{K}$ ) the elements of $\mathcal{Y}_{b}^{x}$ are the admissible portfolio processes.

To use classical stochastic calculus we shall operate with the following right-continuous adapted process of bounded variation

$$
Y_{+}:=Y^{c}+Y^{d}+Y^{d,+},
$$

and use the relation $Y_{+}=Y+\Delta^{+} Y$. Since the generators are right-continuous, the process $Y_{+}$inherits the boundedness from below of $Y$ (by the same constant process $\left.\kappa_{Y} \mathbf{1}\right)$. Note that $\left\|Y_{+}\right\|_{t}=\|Y\|_{t-}+\left|\Delta Y_{t}+\Delta^{+} Y_{t}\right|$.

In the sequel we shall use a larger set portfolio processes depending on $Z \in \mathcal{M}_{0}^{T}\left(G^{*}\right)$, namely,

$$
\mathcal{Y}_{b}^{x}(Z):=\left\{Y \in \mathcal{Y}^{x}: \text { there is a scalar martingale } M \text { such that } Z Y \geq M\right\} .
$$

Lemma 3.1 If $Z \in \mathcal{M}_{0}^{T}\left(G^{*}\right)$ and $Y \in \mathcal{Y}_{b}^{x}(Z)$, then both processes $Z Y_{+}$and $Z Y$ are supermartingales and

$$
E\left(-Z \dot{Y}^{c} \cdot\left\|Y^{c}\right\|_{T}-\sum_{s \leq T} Z_{s-} \Delta Y_{s}-\sum_{s<T} Z_{s} \Delta^{+} Y_{s}\right) \leq Z_{0} x-E Z_{T} Y_{T}
$$

Proof. With the right-continuous process $Y_{+}$(having the same left limits as $Y)$ the standard product formula is readily applied:

$$
Z_{t} Y_{t+}=Z_{0} x+Y_{-} \cdot Z_{t}+Z \dot{Y}^{c} \cdot\left\|Y^{c}\right\|_{t}+\sum_{s \leq t} Z_{s} \Delta Y_{s}+\sum_{s \leq t} Z_{s} \Delta^{+} Y_{s}
$$

Taking into account that $Y=Y_{-}+\Delta Y$, we rewrite this identity as

$$
Z_{t} Y_{t+}=Z_{0} x+Y \cdot Z_{t}+Z \dot{Y}^{c} \cdot\left\|Y^{c}\right\|_{t}+\sum_{s \leq t} Z_{s-} \Delta Y_{s}+\sum_{s \leq t} Z_{s} \Delta^{+} Y_{s}
$$

2 Since $Y$ is a predictable process, the set $\{\Delta Y \neq 0\}$ can be represented as a disjoint union of graphs of predictable stopping times. Hence, 3) implies that $\Delta Y_{\tau} \in-G_{\tau-}$ a.s. for all stopping times $\tau \leq T$. 
Since $Y_{+}=Y+\Delta^{+} Y$, we obtain from here the product formula for $Z Y$ (which is "non-standard" since $Y$ may not be càdlàg):

$$
Z_{t} Y_{t}=Z_{0} x+Y \cdot Z_{t}+Z \dot{Y}^{c} \cdot\left\|Y^{c}\right\|_{t}+\sum_{s \leq t} Z_{s-} \Delta Y_{s}+\sum_{s<t} Z_{s} \Delta^{+} Y_{s}
$$

By virtue of requirements on $Y$ the stochastic integral $Y \cdot Z$ is a local martingale while the last three terms define decreasing processes (by our standing assumption $\left.Z_{s-} \in\left(G_{s-}\right)^{*}\right)$. Recalling that the process $Z Y$ is bounded from below by a martingale, we deduce from here that the local martingale $Y \cdot Z$ is bounded from below by a martingale and, hence, is a supermartingale, hence integrable. It follows that the terminal values of the mentioned decreasing processes are integrable. Therefore, $Z Y$ is a supermartingale. By the Fatou lemma its right-continuous limit, i.e. the process $Z Y_{+}$is a supermartingale. Finally, taking the expectation of the last identity above and using the inequality $Y \cdot Z_{T} \leq 0$, we get the required bound (3.1).

Lemma 3.2 Suppose that $Y^{n} \in \mathcal{Y}$ and for all $\omega$ (except of a null set) $\lim _{n} Y_{t}^{n}(\omega)=Y_{t}(\omega)$ for all $t \in[0, T]$, where $Y$ is a process of bounded variation. Then the process $Y$ belongs to $\mathcal{Y}$.

This assertion follows immediately from the alternative description of $\mathcal{Y}$ given in the lemma below.

Lemma 3.3 Let $Y$ be a predictable process of bounded variation. Then

$$
Y \in \mathcal{Y} \Leftrightarrow Y_{\sigma}-Y_{\tau} \in L^{0}\left(G_{\sigma, \tau}\right) \text { for all stopping times } \sigma, \tau, \sigma \leq \tau \leq T \text {. }
$$

Proof. $(\Rightarrow)$ Follows obviously from the representation

$$
Y_{\tau}-Y_{\sigma}=\int_{\sigma}^{\tau} \dot{Y}_{r}^{c} d\left\|Y^{c}\right\|_{r}+\sum_{\sigma<r \leq \tau} \Delta Y_{r}+\sum_{\sigma \leq r<\tau} \Delta^{+} Y_{r} .
$$

$(\Leftarrow)$ First, we provide an "explicit" formula for $\dot{Y}^{c}$ using the classical approach due to Doob, see [6], V.5.58. For the reader's convenience we recall the idea. Put $t_{k}=t_{k}^{n}=k 2^{-n} T$, fix $\omega$ (omitted in the notation) and consider the sequence of functions

$$
X_{n}(t)=\sum_{k} \frac{Y_{t_{k+1}^{n}+}-Y_{t_{k}^{n}+}}{\left\|Y_{+}\right\|_{t_{k+1}^{n}}-\left\|Y_{+}\right\|_{t_{k}^{n}}} I_{] t_{k}^{n}, t_{k+1}^{n}\right]}(t), \quad[0, T] .
$$

This sequence is a bounded martingale with respect to the dyadic filtration on $[0, T]$ and the finite measure $d\left\|Y_{+}\right\|$. So, it converges (almost everywhere with respect to this measure) to a limit $X_{\infty}$ which is the Radon-Nikodym derivative $d Y_{+} / d\left\|Y_{+}\right\|$and which may serve also as the Radon-Nikodym derivative $d Y^{c} / d\left\|Y^{c}\right\|$.

Thus,

$$
\dot{Y}^{c}=\limsup _{n} \sum_{k} \frac{Y_{t_{k+1}^{n}+}-Y_{t_{k}^{n}+}}{\left\|Y_{+}\right\|_{t_{k+1}^{n}}-\left\|Y_{+}\right\|_{t_{k}^{n}}^{n}} I_{\left.t_{k}^{n}, t_{k+1}^{n}\right]} \quad d P d\left\|Y^{c}\right\| \text {-a.e. }
$$


It follows that $-\dot{Y}_{t}^{c} \in G_{t-, t+}$ (a.e.). By assumption, $G_{t-, t+}=\operatorname{cone}\left\{G_{t-}, G_{t}\right\}$. But for each $\omega$ the set $\left\{t: G_{t}(\omega) \neq G_{t-}(\omega)\right\}$ is at most countable and the property 1) in the definition of $\mathcal{Y}$ is fulfilled.

For a stopping time $\tau$ we put $\tau^{n}:=\tau+1 / n$. Then $\tau_{n} \downarrow \tau$

$$
\Delta^{+} Y_{\tau}=\lim _{n}\left(Y_{\tau^{n}}-Y_{\tau}\right) \in-G_{\tau, \tau+}=-G_{\tau} .
$$

For a predictable stopping time $\sigma$ one can find an announcing sequence of stopping times $\sigma^{n} \uparrow \sigma$ with $\sigma^{n}<\sigma$ on the set $\{\sigma>0\}$. Thus, on this set

$$
\Delta Y_{\sigma}=\lim _{n}\left(Y_{\sigma}-Y_{\sigma^{n}}\right) \in-G_{\sigma-, \sigma}=-G_{\sigma-} .
$$

The lemma is proven.

Lemma 3.4 Let $Z \in \mathcal{D}$. Let $A$ be a subset of $\mathcal{Y}_{b}^{0}(Z)$ for which there is a constant $\kappa$ such that $Y_{T}+\kappa \mathbf{1} \in L^{0}\left(G_{T}, \mathcal{F}_{T}\right)$ for all $Y \in A$. Then there exists a probability measure $Q \sim P$ such that $\sup _{Y \in A} E_{Q}\|Y\|_{T}<\infty$.

Proof. Fix $Z \in \mathcal{D}$ and consider the random variable

$$
\alpha:=\inf _{t \leq T} \inf _{x \in G_{t},|x|=1} Z_{t} x=\inf _{t \leq T} Z_{t} x_{t},
$$

where $x_{t}=x_{t}(\omega)$ is the point on the unit sphere at which the interior infimum is attained. If $t_{n} \downarrow t_{0}$ and the sequence $x_{t_{n}}$ tends to some $x_{0}$, then the point $x_{0} \in \cap_{\varepsilon>0} G_{t_{0}, t_{0}+\varepsilon}=G_{t_{0}, t_{0}+}$. By our assumption, $G_{t_{0}, t_{0}+}=G_{t_{0}}$. If $t_{n} \uparrow t_{0}$ and the sequence $x_{t_{n}}$ tends to some $x_{0}$, then $x_{0} \in G_{t_{0}-}$ by virtue of a similar argument. On various $\omega$ the infimum in $t$ can be obtained either on a decreasing sequence of $t_{n}$ (in this case, $\alpha=Z_{t_{0}} x_{t_{0}}$ ) or on a increasing one (in this case, $\alpha=Z_{t_{0}-} x_{t_{0}}$ ). The assumption on $Z$ guaranties that in both cases the values of $\alpha$ are strictly positive.

It is easily seen that the left-hand side of (3.1) dominates

$$
E \alpha\left(\left|\dot{Y}^{c}\right| \cdot|| Y^{c} \|_{T}+\sum_{s \leq T}\left|\Delta Y_{s}\right|+\sum_{s<T}\left|\Delta^{+} Y_{s}\right|\right)=E \alpha\|Y\|_{T}
$$

and, therefore,

$$
E \alpha e^{-\alpha}\|Y\|_{T} \leq E \alpha\|Y\|_{T} \leq Z_{0} x-E Z_{T} Y_{T} \leq Z_{0} x+\kappa E Z_{T} \mathbf{1} .
$$

It follows that the measure $Q$ with the density $d Q / d P=\alpha e^{-\alpha} /\left(E \alpha e^{-\alpha}\right)$ is the required one.

Recall that a sequence $a^{n}$ is Césaro convergent if $\bar{a}^{n}:=n^{-1} \sum_{k=1}^{n} a^{k}$ converges. The Komlós theorem asserts that if $\xi^{n}$ are random variables with $\sup _{n} E\left|\xi^{n}\right|<\infty$ then there exist $\xi \in L^{1}$ and a subsequence $\xi^{n^{\prime}}$ such that all its subsequences are Césaro convergent to $\xi$ a.s.

Lemma 3.5 Let $A^{n}$ be a sequence of predictable increasing processes starting from zero and with $\sup _{n} E A_{T}^{n}<\infty$. Then there is an increasing process $A$ with $A_{T} \in L^{1}$ and a subsequence $A^{n^{\prime}}$ which is Césaro convergent to $A$ pointwise at every point of $[0, T]$ for all $\omega$ except a P-null set. 
Proof. Let $\mathbf{T}:=\left\{k 2^{-n} T: k=0, \ldots, 2^{n}, n \in \mathbf{N}\right\}$. Using the Komlós theorem and the diagonal procedure we find a subsequence such that $A_{r}^{n^{\prime}}$ is Cesaro convergent a.s. to $A_{r}^{o} \in L^{1}$ (with all further subsequences) for all $r \in \mathbf{T}$. We can always choose a common null set $\Omega_{0}$ and assume that $A_{r}^{o}(\omega)$ is increasing in $r$ for each $\omega \notin \Omega_{0}$. Let us consider its left-continuous envelope, defined on the whole interval, i.e. the process $A_{t}:=\liminf _{r \uparrow t} A_{t}^{o}(r \in \mathbf{T}$ and $r<t)$. By the same argument as in the theory of weak convergence of probability distribution functions, we conclude that if the sequence of functions $A^{n^{\prime}}(\omega)$ converges at all points of $\mathbf{T}$ in Césaro sense to $A^{o}(\omega)$, then it converges, in the same sense, to the function $A(\omega)$ at all points of continuity of the latter. The crucial observation is that one can sacrifice the left-continuity of $A$ but "improve" the convergence property. To this aim let us consider a sequence of stopping times $\tau_{k}$ exhausting the jumps of the process $A$ (i.e. such that $\left\{\Delta^{+} A>0\right\} \subseteq \cup_{k}\left[\tau_{k}\right]$ where $\left[\tau_{k}\right]$ is the graph of $\left.\tau_{k}\right)$. Refining the subsequence of $A^{n^{\prime}}$ we may assume that each sequence of random variables $A_{\tau_{k}}^{n^{\prime}}$ also converges in Césaro sense. Replacing $A_{\tau_{k}}$ by these limiting values we obtain the required process which is a pointwise Césaro limit of a certain subsequence of $A^{n}$ (thus, predictable).

Remark. The above lemma from [3] is the key element of our proof and it merits to be well-understood. It is worthy to make a look at its deterministic counterpart which is just a version of the Helly theorem. The latter is usually formulated for left-continuous (or, more frequently, for right-continuous) monotone functions. The proof is easy: combining the Bolzano-Weierstrass theorem and the diagonal procedure one defines a monotone function $A^{\circ}$ on $\mathbf{T}$ and a subsequence $A^{n^{\prime}}$ convergent to $A^{o}$ on $\mathbf{T}$. Let $A$ be the left envelope of $A^{\circ}$. Due to monotonicity, the same subsequence will converge to all points of $[0, T]$ where $A$ is continuous and this gives the standard version of the Helly theorem. Of course, the convergence may fail at the denumerable set where $A$ is discontinuous. Repeating the arguments, one can find a further subsequence having limits also at each point of discontinuity of $A$. Replacing the values of $A$ by these limits, we get an increasing function approximated by the refined subsequence at all points of the interval. The proof in the stochastic setting follows the same lines with the classical compactness argument replaced by a reference to the Komlós theorem.

\section{Hedging of American options}

Let $U=\left(U_{t}\right)_{t \in[0, T]}$ be an $\mathbf{R}^{d}$-valued càdlàg process for which there is a constant $\kappa$ such that $U_{t}+\kappa \mathbf{1} \in L^{0}\left(G_{t}, \mathcal{F}_{t}\right)$ for all $t$. In the context of financial modelling such a process is interpreted as (the pay-off of) an American option. Let $A_{0}^{T}$ (.) be the set of American options which can be dominated by a portfolio with zero initial capital, i.e. by an element of $\mathcal{Y}_{b}^{0}$.

Define the convex set

$\Gamma:=\left\{x \in \mathbf{R}^{d}: \exists Y \in \mathcal{Y}_{b}^{x}\right.$ such that $\left.Y \geq_{G} U\right\}=\left\{x \in \mathbf{R}^{d}: U-x \in A_{0}^{T}().\right\}$, and the closed convex set

$$
D:=D(P):=\left\{x \in \mathbf{R}^{d}: \bar{Z}_{0}^{\nu} x \geq E^{\nu} Z U \quad \forall Z \in \mathcal{Z}\left(G^{*}, P, \nu\right), \forall \nu \in \mathcal{N}\right\} .
$$


It is easy to check that $D(P)=D(\tilde{P})$ if $\tilde{P} \sim P$. Indeed, let $x \in D(P)$, $\nu \in \mathcal{N}$, and $\tilde{Z} \in \mathcal{Z}\left(G^{*}, \tilde{P}, \nu\right)$. Define $\rho_{t}=E\left(d \tilde{P} / d P \mid \mathcal{F}_{t}\right)$ and consider the process $Z_{t}=\rho_{t} \tilde{Z}_{t}$. It is in $\mathcal{Z}\left(G^{*}, P, \nu\right)$ and

$$
E \rho_{T} \int_{[0, T]} \tilde{Z}_{t} U_{t} \nu(d t)=E \int_{[0, T]} \rho_{t} \tilde{Z}_{t} U_{t} \nu(d t)=E \int_{[0, T]} Z_{t} U_{t} \nu(d t) \leq x E^{\nu} Z
$$

Since $E^{\nu} Z=\tilde{E}^{\nu} \tilde{Z}$, it follows that $x \in D(\tilde{P})$.

Proposition 4.1 $\Gamma \subseteq D$.

Proof. Let $x \in \Gamma$. Then there exists $Y$ in $\mathcal{Y}_{b}^{0}$ such that the process $x+Y$ dominates $U$, i.e. $x+Y_{t}-U_{t} \in L^{0}\left(G_{t}, \mathcal{F}_{t}\right)$ for all $t \in[0, T]$. It follows that $x+Y_{t+}-U_{t} \in L^{0}\left(G_{t}, \mathcal{F}_{t}\right)$. By duality, for any $Z \in \mathcal{Z}\left(G^{*}, P, \nu\right)$ and $\nu \in \mathcal{N}$ we have that

$$
E \int_{[0, T]} Z_{t} x \nu(d t)+E \int_{[0, T]} Z_{t} Y_{t+} \nu(d t) \geq E \int_{[0, T]} Z_{t} U_{t} \nu(d t) .
$$

It remains to verify that $E^{\nu} Z Y_{+} \leq 0$. Using the Fubini theorem and the property of the optional projection given by Th.VI.2.57 in [6] we have:

$$
\begin{aligned}
E \int_{[0, T]} Z_{t} Y_{t+} \nu(d t) & =E \int_{[0, T]} Z_{t}\left(\int_{[0, t]} \dot{Y}_{+s} d\left\|Y_{+}\right\|_{s}\right) \nu(d t) \\
& =E \int_{[0, T]} \dot{Y}_{+s}\left(\int_{[s, T]} Z_{t} \nu(d t)\right) d\left\|Y_{+}\right\|_{s} \\
& =E \int_{[0, T]} \dot{Y}_{+s} \bar{Z}_{s}^{\nu} d\left\|Y_{+}\right\|_{s}
\end{aligned}
$$

It is easy to see that

$$
\int_{[0, T]} \dot{Y}_{+s} \bar{Z}_{s}^{\nu} d\left\|Y_{+}\right\|_{s}=\int_{[0, T]} \dot{Y}_{s}^{c} \bar{Z}_{s}^{\nu} d\left\|Y^{c}\right\|_{s}+\sum_{s \leq T} \bar{Z}_{s}^{\nu} \Delta Y_{s}+\sum_{s \leq T} \bar{Z}_{s}^{\nu} \Delta^{+} Y_{s} .
$$

Since $\dot{Y}_{s}^{c}$ and $\Delta^{+} Y_{s}$ take values in the cone $-G_{s}$ and $\bar{Z}_{s}^{\nu}$ takes values in $G_{s}^{*}$, the first and the third terms of the above identity are negative. The increment $\Delta Y_{s}$ takes values in $-G_{s-}$. If $G_{s-}=G_{s}$ for all $s$, the second term is also negative and we conclude. As we do not assume the continuity of the process $G$, the proof requires a bit more work.

Let us suppose for a moment that the random variable $\left\|Y^{d}\right\|_{T}$ is bounded. To get the needed inequality $E^{\nu} Z Y_{+} \leq 0$ it is sufficient to check that the expectation of the second term is negative. We proceed as follows. Recall that $\bar{Z}^{\nu}=M^{\nu}-R$ where $M^{\nu}$ is a martingale and the process

$$
R_{t}=\int_{[0, t[} Z_{u} \nu(d u)
$$


is left-continuous. The last property implies that $\Delta \bar{Z}^{\nu}=\Delta M^{\nu}$. It follows that

$$
\sum_{s \leq T} \bar{Z}_{s}^{\nu} \Delta Y_{s}=\sum_{s \leq T} \bar{Z}_{s-}^{\nu} \Delta Y_{s}+\sum_{s \leq T} \Delta M_{s}^{\nu} \Delta Y_{s} .
$$

The first sum in the right-hand side is obviously negative while the expectation of the second one is zero. This follows from the classical property (see, e.g. [7], Lemma I.3.12): if $M$ is a positive martingale and $B$ is a predictable increasing process starting from zero, then

$$
E M_{T} B_{T}=E \int_{[0, T]} M_{s} d B_{s}=E \int_{[0, T]} M_{s-} d B_{s} .
$$

We can easily remove the condition of the boundedness of $\left\|Y^{d}\right\|$. Indeed, a finite predictable increasing process is locally bounded, see [6], Ch. VIII.11. Hence, there is a sequence of stopping times $\tau^{n}$ increasing stationary to $T$ (i.e. with $\left.P\left(\tau_{n}=T\right) \rightarrow 1\right)$ such that $\left\|Y^{d}\right\|_{\tau^{n}} \leq C_{n}$. Let $U^{n}$ be a process coinciding with $U$ on $\left[0, \tau^{n}\right.$ [ and taking the value $x+Y_{\tau^{n}}$ on $\left[\tau^{n}, T\right]$. It follows from the above arguments that $\bar{Z}^{\nu} x \geq E^{\nu} Z U^{n}$ and the result follows from the Fatou lemma.

Theorem 4.2 Suppose that $\mathcal{D} \neq \emptyset$. Then $\Gamma=D$.

Proof. We fix $\tilde{Z} \in \mathcal{D}$ and define the set of hedging endowments corresponding to portfolios with the "relaxed" admissibility property, namely, we put

$$
\Gamma(\tilde{Z}):=\left\{x \in \mathbf{R}^{d}: \exists Y \in \mathcal{Y}_{b}^{x}(\tilde{Z}) \text { such that } Y \succeq_{G} U\right\} .
$$

Since $\mathcal{Y}_{b}^{x}(\tilde{Z}) \supseteq \mathcal{Y}_{b}^{x}$, this set is larger than $\Gamma$. On the other hand, if a portfolio $Y$ dominate $U$, it is bounded from below. Hence, $\Gamma(\tilde{Z})=\Gamma$.

Let $\mathbf{T}^{m}:=\left\{t_{k}=t_{k}^{m}: t_{k}=k 2^{-m} T, k=0, \ldots, 2^{m}\right\}$; then $\mathbf{T}=\cup_{m \geq 1} \mathbf{T}^{m}$. Define the convex set $A_{\mathbf{T}^{m}}($.$) of American options W$ which can be hedged at the dates from $\mathbf{T}^{m}$ by a portfolio belonging to the class $\mathcal{Y}_{b}^{0}(\tilde{Z})$, i.e. such that $Y_{t}-W_{t} \in G_{t}, t \in \mathbf{T}^{m}$, for some $Y \in \mathcal{Y}_{b}^{0}(\tilde{Z})$. Let us consider $A_{\mathbf{T}^{m}}($.$) as$ a subset of the space $L^{0}\left(P \otimes \nu^{m}\right):=L^{0}\left(\Omega \times[0, T], \mathcal{F} \times \mathcal{B}_{[0, T]}, P \otimes \nu^{m}\right)$ where the probability measure $\nu^{m}$ is the uniform distribution on $\mathbf{T}^{m}$, i.e. it charges only the points of $\mathbf{T}^{m}$ with weights $1 /\left(2^{m}+1\right)$. From the point of view of this space, $W$ is just the random vector $\left(W_{0}, W_{1 / 2^{m}}, \ldots, W_{T}\right)$ (the components of the latter are $d$-dimensional). For such random vectors (with fixed $m \geq 1$ ) we extend the concept of the Fatou-convergence in the same spirit as was developed in the problem of hedging of European options. Note that $A_{\mathbf{T}^{m}}($.$) ,$ in general, depends on $\tilde{Z}$.

We say that a sequence $W^{n}$ is Fatou-convergent in $L^{0}\left(P \otimes \nu^{m}\right)$ to $W$ if there is a constant $\kappa$ such that $W_{r}^{n}+\kappa \mathbf{1} \in L^{0}\left(G_{r}, \mathcal{F}_{r}\right)$ (i.e., $W_{r}^{n} \succeq_{G_{r}}-\kappa \mathbf{1}$ ) for all $r \in \mathbf{T}^{m}, n \geq 1$, and $W_{r}^{n} \rightarrow W_{r}$ a.s., $n \rightarrow \infty$, for all $r \in \mathbf{T}^{m}$. The subsequent definitions Fatou-closed and Fatou-dense are obvious.

Lemma 4.3 The set $A_{\mathbf{T}^{m}}($.$) is Fatou-closed in L^{0}\left(P \otimes \nu^{m}\right)$. 
Proof. Let $W^{n} \in A_{\mathbf{T}^{m}}$ (.) be a sequence Fatou-converging to $W$ and let $Y^{n}$ be a corresponding sequence of dominating elements from $\mathcal{Y}_{b}^{0}(\tilde{Z})$. Our aim is to show that $W$ also can be dominated by some element $\mathcal{Y}_{b}^{0}(\tilde{Z})$ at the points of $\mathbf{T}^{m}$. Using the preceding results (see Lemmas 3.4 and 3.5) we can replace $W^{n}$ and $Y^{n}$ by appropriate sequences of arithmetic means and suppose without loss of generality that $Y^{n}$ converges to some predictable process $Y$ of bounded variation almost surely at each point $t \in[0, T]$. Using Lemma 3.2 we conclude that $Y \in \mathcal{Y}^{0}$. It remains to check that $\tilde{Z} Y$ dominates a martingale. By virtue of Lemma 3.1 the prelimit processes $\tilde{Z} Y^{n}$ are supermartingales. Since $Y_{T}^{n}$ dominates $W_{T} \succeq_{G_{T}}-\kappa \mathbf{1}$, we have that $\tilde{Z}_{T} Y_{T}^{n} \geq-\kappa \tilde{Z}_{T}$. It follows that the supermartingale $\tilde{Z} Y^{n}$ dominates the martingale $-\kappa \tilde{Z}$ and so does the supermartingale $\tilde{Z} Y$.

Lemma 4.4 The set $A_{\mathbf{T}^{m}}(.) \cap L^{\infty}\left(P^{\nu^{m}}\right)$ is Fatou-dense in $A_{\mathbf{T}^{m}}($.$) .$

Proof. Let $W \in A_{\mathbf{T}^{m}}($.$) be dominated at the points of \mathbf{T}^{m}$ by a portfolio $Y \in \mathcal{Y}_{b}^{0}(\tilde{Z})$. Let $\kappa$ be a constant such that $W_{t}+\kappa \mathbf{1} \in G_{t}$ for $t \in \mathbf{T}^{m}$. Put

$$
W^{n}:=W I_{\{|W| \leq n\}}-\kappa I_{\{|W|>n\}} .
$$

Then $W^{n} \in L^{\infty}\left(P \otimes \nu^{m}\right)$ and tends to $W$ as $n \rightarrow \infty$. Since

$$
Y_{t}-W_{t}^{n}=\left(Y_{t}-W_{t}\right) I_{\left\{\left|W_{t}\right| \leq n\right\}}+\left(Y_{t}+\kappa \mathbf{1}\right) I_{\left\{\left|W_{t}\right|>n\right\}} \in G_{t}, \quad t \in \mathbf{T}^{m},
$$

$W^{n} \in A_{\mathbf{T}^{m}}($.$) .$

Let $L_{b}^{0}\left(P \otimes \nu^{m}\right)$ be the cone in $L^{0}\left(P \otimes \nu^{m}\right)$ formed by the elements $W$ (interpreted as random vectors) which are adapted and bounded from below in the sense of partial ordering, i.e. such that $W_{r}+c \mathbf{1} \in L^{0}\left(G_{r}, \mathcal{F}_{r}\right)$ for all $r \in \mathbf{T}^{m}$. The notation $L^{1}\left(G^{*}, P \otimes \nu^{m}\right)$ has an obvious meaning.

The following lemma is Theorem 4.3 from [11] formulated in the notation adjusted to the considered situation (where one take $W_{0}=0$ ).

Lemma 4.5 Let $A$ be a convex subset in $L_{b}^{0}\left(P \otimes \nu^{m}\right)$ which is Fatou-closed and such that the set $A^{\infty}:=A \cap L^{\infty}\left(\mathbf{R}^{d}, P \otimes \nu^{m}\right)$ is Fatou-dense in $A$. Suppose that there is $W_{0} \in A^{\infty}$ such that $W_{0}-L^{\infty}\left(G, P \otimes \nu^{m}\right) \subseteq A^{\infty}$. Then

$$
A=\left\{W \in L_{b}^{0}\left(P \otimes \nu^{m}\right): E^{\nu^{m}} Z W \leq f(Z) \forall Z \in L^{1}\left(G^{*}, P \otimes \nu^{m}\right)\right\}
$$

where $f(Z)=\sup _{Y \in A} E^{\nu^{m}} Z Y$.

With the above preliminaries we can complete the proof of Theorem 4.2 by establishing the remaining inclusion $D \subseteq \Gamma=\Gamma(\tilde{Z})$. Indeed, take a point $x \in D$. Suppose that $U-x \notin A_{\mathbf{T}^{\mathrm{m}}}($.$) for some m$. By virtue of Lemma 4.5 there exists $Z \in L^{1}\left(G^{*}, P \otimes \nu^{m}\right)$ such that $E^{\nu^{m}} Z(U-x)>f(Z)$. But $f(Z)=0$ as $A_{\mathbf{T}^{\mathrm{m}}}($.$) is a cone. We can identify Z$ with a right-continuous adapted process taking value $Z_{t_{k}}$ at the points $t_{k}$. Since $E^{\nu^{m}} Z Y \leq 0$ for all $Y \in A_{\mathbf{T}^{\mathrm{m}}}($.$) , the process Z \in \mathcal{Z}\left(G^{*}, P, \nu^{m}\right)$. Thus, $x \notin D$, a contradiction. This means that $U-x \in A_{\mathbf{T}^{m}}($.$) for all m$, i.e. there exist admissible portfolio processes $Y^{n}$ dominating $U-x$ at the points of $\mathbf{T}^{n}$. In particular, the sequence $Y_{T}^{n}$ is bounded from below by a constant vector and, by virtue of Lemma 3.4, 
the total variations $\left\|Y^{n}\right\|_{T}$ are bounded in a certain $L^{1}(Q)$ with $Q \sim P$. Using Lemma 3.5, we may assume without loss of generality that the sequence $Y^{n}$ converges to some predictable process $Y$ of bounded variation almost surely at each point $t \in[0, T]$. Recall that $U_{t}+\kappa \mathbf{1} \in G_{t}$. The limiting process $Y$ dominates $U-x$ at all points from $\mathbf{T}$. Using the right continuity of the processes, we obtain that $Y_{+}$dominates $U-x$ on the whole interval and so does the "larger" process $Y$. So, $x \in \Gamma$.

Remark. Theorem 4.2 implies as a corollary a hedging theorem for càdlàg portfolio processes under assumption that the cone-valued process $G$ is continuous. Indeed, let $\mathcal{X}^{0}$ be the set of all càdlàg processes $X$ of bounded variation with $X_{0}=0$ and such that $d X / d\|X\| \in-G d P d\|X\|$-a.e. The notations $\mathcal{X}^{x}$ and $\mathcal{X}_{b}^{x}$ are obvious. Let

$$
\Gamma_{X}:=\left\{x \in \mathbf{R}^{d}: \exists X \in \mathcal{X}_{b}^{x} \text { such that } X \geq_{G} U\right\} .
$$

Similar (but simpler) arguments than that used in the proof of Proposition 4.1 show that $\Gamma_{X} \subseteq D$.

Suppose that all generators $\xi^{k}$ of $G$ are continuous processes. It is easy to check that if the process $Y \in \mathcal{Y}^{0}$ then $Y_{+} \in \mathcal{X}^{0}$. Thus, $\Gamma \subseteq \Gamma_{X}$ and Theorem 4.2 implies that if $\mathcal{D}(G) \neq \emptyset$, then $\Gamma_{X}=D$.

\section{Financial Interpretation: Coherent Price Systems}

In the final section of this note we want to attract the reader's attention to the financial interpretation of the obtained result. In the hedging theorems for European options the important concept is a consistent price system which replaces the notion of the martingale density of the classical theory sometimes referred to as "stochastic deflator" or "state-price density". The words "price system" mean that it is a process evolving in the duals $\widehat{K}_{t}^{*}$ to the solvency cones $\widehat{K}_{t}$ while "consistent" alludes that this process is a martingale. Hedging theorems are results claiming that a contingent claim $\xi$ (in physical units) can be super-replicated starting from an initial endowment $x$ by a self-financing portfolio if and only if the "value" $Z_{0} x$ of this initial endowment is not less than the expected "value" of the contingent claim $E Z_{T} \xi$ for any consistent price system $Z$ (we write the word "value" in quotation marks to emphasize its particular meaning in the present context). In other words, consistent price systems allow the option seller to relate benefits from possessing $x$ at time $t=0$ and the liabilities $\xi$ at time $t=T$ and provide information whether there is a portfolio ending up on the safe side.

The situation with the American option is different. As it was observed by Chalasani and Jha, already in the simplest discrete-time models consistent price systems form a class which is too narrow to evaluate American claims correctly. The phenomenon appears because one cannot prohibit the option buyer to toss a coin and take a decision, to exercise or not, in dependence of the outcome. A financial intuition suggests that the expected "value" of an American claim is an expectation of the weighted average of "values" of assets obtained by the option holder for a variety of exercise dates. This expected 
"value" should be compared with the "value" of the initial endowment. The main question is: what is the class of price systems which should be involved to compute the "values" to be compared? Our result shows, that in a rather general continuous-time model, the comparison can be done with the systems for which the expected weighted average of future prices knowing the past is again a price system. The structure of such a price system is coherent with the option buyer actions and we propose to call it coherent price system and use the abbreviation CoPS.

It is well-known that without transaction costs the rational exercise strategy of the buyer is the optimal solution of a stopping problem which exists in the class of pure stopping times. This explains why in the models of frictionless markets there is no need to go beyond the class of consistent price systems. For markets with transaction costs the rational exercise strategies of the option buyer is an open problem.

A reader acquainted with set-valued analysis may ask a question why we limit ourselves by considering a rather particular cone-valued process defined via a countable family of generators. Indeed, it seems that the natural mathematical framework is a model given by a general cone-valued process $G$ satisfying certain continuity conditions. A possible generalizations of this kind and a development of the theory of set-valued processes are of interest and can be subjects of further studies.

Remark. To the present, the pay-off of American options was usually modelled by a right-continuous (or left-continuous) process. Though we believe that this class is sufficient for financial applications, the problem of the dual description of the set of hedging endowments for the processes only admitting right and left limits is mathematically interesting. It is solved in the preprint [1] by Bouchard and Chassagneux which appeared when our paper was under refereeing. Their dual variables are different from those introduced here and the relations between two descriptions are left by the authors of [1] as a subject of further studies.

Acknowledgements The authors express their thanks to anonymous referees for helpful suggestions to improve the presentation.

\section{References}

1. Bouchard B., Chassagneux J.-F. Representation of continuous linear forms on the set of ladlag processes and the pricing of American claims under proportional costs. Preprint.

2. Bouchard B., Temam E. On the hedging of American options in discrete time markets with proportional transaction costs. Electronic J. Probability, 10 (2005), 22, 746-760 (electronic).

3. Campi L., Schachermayer W. A super-replication theorem in Kabanov's model of transaction costs. Finance and Stochastics, 10 (2006), 4, 579-596.

4. Chalasani P., Jha S. Randomized stopping times and American option pricing with transaction costs. Mathematical Finance, 11 (2001), 1, 33-77.

5. Cvitanić J., Karatzas I. Hedging and portfolio optimization under transaction costs: a martingale approach. Mathematical Finance, 6 (1996), 2, 133-165.

6. Dellacherie C., Meyer P.-A. Probabilités et Potenciel. Hermann, Paris, 1980. 
7. Jacod J., Shiryaev A.N. Limit Theorems for Stochastic Processes. Springer, Berlin-Heidelberg-New York, 1987.

8. Kabanov Yu.M. Hedging and liquidation under transaction costs in currency markets. Finance and Stochastics, 3 (1999), 2, 237-248.

9. Kabanov Yu.M. Arbitrage theory. In: Handbooks in Mathematical Finance. Option Pricing: Theory and Practice. Cambridge University Press, 2001, 3-42.

10. Kabanov Yu.M., Last G. Hedging under transaction costs in currency markets: a continuous-time model. Mathematical Finance, 12 (2002), 1, 63-70.

11. Kabanov Yu.M., Stricker Ch. Hedging of contingent claims under transaction costs. Advances in Finance and Stochastics. Eds. K. Sandmann and Ph. Schönbucher, Springer, 2002, 125-136.

12. Kramkov D.O. Optional decomposition of supermartingales and hedging in incomplete security markets. Probability Theory and Related Fields, 105 (1996), 4, 459-479.

13. Rásonyi M. A remark on the superhedging theorem under transaction costs. Séminaire de Probabilités XXXVII, Lecture Notes in Math., 1832, Springer, Berlin-Heidelberg-New York, 2003, 394-398. 